\title{
American Institute of Ultrasound in Medicine Recommendations for Contrast-Enhanced Liver Ultrasound Imaging Clinical Trials
}

\author{
Lennard Greenbaum, MD, Peter Burns, PhD, Joshua Copel, MD, \\ David Cosgrove, MD, J. Brian Fowlkes, PhD, Barry Goldberg, MD, \\ Robert Mattrey, MD, Daniel Merton, BS, RDMS, Michelle Robbin, MD, \\ Stephanie Wilson, MD
}

\begin{abstract}
Abbreviations
AlUM, American Institute of Ultrasound in Medicine; ALARA, as low as reasonably achievable; CEUS, contrast-enhanced ultrasound; CT, computed tomographic; FDA, Food and Drug Administration; Ml, mechanical index; MR, magnetic resonance; PVC, premature ventricular contraction
\end{abstract}

Received January 25, 2007, from Orlando Regional Healthcare, Orlando, Florida USA (L.G.); Sunnybrook Health Sciences Center, Toronto, Ontario, Canada (P.B.); Yale University School of Medicine, New Haven, Connecticut USA (J.C.); Hammersmith Hospital, London, England (D.C.); University of Michigan Health System, Ann Arbor, Michigan (J.B.F.); Thomas Jefferson University Hospital, Philadelphia, Pennsylvania USA (B.G., D.M.); University of California San Diego Medical Center, San Diego, California USA (R.M.); University of Alabama at Birmingham, Birmingham, Alabama USA (M.R.); and University of Toronto, Toronto, Ontario, Canada (S.W.). Manuscript accepted for publication January 29, 2007.

These recommendations were developed by the AIUM in consultation with the following companies: Bracco Imaging SpA, Bristol-Myers Squibb Medical Imaging, GE Healthcare, National Electrical Manufacturers Association, Philips Medical Systems Ultrasound, Siemens Medical Solutions, Inc, and SonoSite. The AIUM acknowledges these companies for the background that they provided; it was essential to the development of this document.

Address correspondence to Lennard Greenbaum, MD, Winnie Palmer Hospital for Women \& Babies, The Hughes Center for Fetal Diagnostics, 83 W Miller St, Orlando, FL 32806 USA.

E-mail: lengreenbaum@msn.com

\section{Preamble}

The American Institute of Ultrasound in Medicine (AIUM) is a recognized authority in the clinical utility, education, and safety of ultrasound imaging. The AIUM has many standing committees, including the Clinical Standards Committee, Continuing Medical Education Committee, and Ultrasound Practice Accreditation Council. The Bioeffects Committee monitors the safety of ultrasound and contrast agents on a continuing basis. ${ }^{1,2}$

As a multispecialty, clinically oriented organization of ultrasound specialists, the AIUM participates closely in developments that it identifies as essential for the medical community to improve patient care. The introduction of ultrasound contrast media in the mid-1990s has already revolutionized the practice of ultrasound imaging in those countries where they are available. In particular, it has been established that ultrasound imaging performed with contrast enhancement (contrast-enhanced ultrasound [CEUS]) allows for accurate characterization and detection of focal liver lesions, which often are not possible with conventional ultrasound imaging. Numerous single- and multi-center investigations of CEUS have shown that both detection and characterization of liver masses are comparable to those achieved with contrast-enhanced computed tomographic (CT) or magnetic resonance (MR) scans. Ultrasound imaging performed with contrast enhancement is clearly superior to ultrasound imaging performed alone. In fact, the lack of specificity for the confident diagnosis of liver masses with ultrasound imaging alone is so well recognized that, 
in most cases, the identification of a liver mass on ultrasound imaging provokes confirmatory imaging with either a CT or an MR scan. In patients at risk for hepatocellular carcinoma or metastases, there is recognition that confirmation of all masses identified on ultrasound imaging with either CT or MR scans is essential.

In addition to the lack of specificity for ultrasound-based diagnosis of liver masses, ultrasound-based detection of focal liver masses can be poor, especially in patients at risk for metastatic liver disease. Although some metastases may be easily seen on ultrasound imaging because they are either echogenic or hypoechoic relative to the liver parenchyma, many others have backscatter similar to that of the background liver. These so-called "isoechoic" or "invisible masses" have backscatter that is identical to that of the adjacent normal parenchyma, and they are difficult if not impossible to detect without the aid of ultrasound contrast. Studies have shown that these masses can be identified with a sensitivity comparable to that of contrastenhanced CT or MR imaging by the addition of ultrasound contrast agents. A review of the world literature on the efficacy of CEUS imaging compared with non-CEUS imaging for the detection and characterization of liver lesions is documented in "Appendix A."

A key element in the evolution of medical imaging technology has been the development from plain imaging to techniques enhanced by intravascular contrast agents. In many countries outside the United States, it is no longer considered sufficient to perform only non-CEUS imaging of the liver for detection and characterization of focal liver masses.

It is the AIUM's considered opinion that the lack of availability of CEUS for noncardiac imaging in the United States hinders the delivery of optimal diagnostic imaging services to our patients. As a result, we lag behind the rest of the world in the appropriate and proven uses of contrast agents for liver mass diagnosis and detection. The AIUM believes that this is having an adverse impact on clinical care in the United States.

Therefore, pursuant to the request by the US Food and Drug Administration (FDA), the AIUM proposes the following recommendations for consideration for future CEUS clinical trials.

\section{Appropriate End Points for Assessment of Ultrasound Contrast Efficacy in the Liver}

Ultrasound imaging of the liver in the United States is currently performed without contrast media. Contrast media are intended to improve lesion conspicuity for increased lesion detection sensitivity and/or to improve lesion characterization for increased diagnostic specificity. Improved detection sensitivity and/or diagnostic specificity when a contrast agent is used compared with conventional non-CEUS imaging should be sufficient to establish efficacy for approval. A recommended trial, therefore, should compare the non-CEUS imaging performance with the CEUS imaging performance against an accepted truth standard. The basis for FDA approval should be a significant improvement of CEUS over non-CEUS imaging.

The AIUM believes that there are situations in which it is clearly obvious that the improved diagnostic/prognostic information achieved with CEUS imaging is clinically useful. These should be considered an adequate basis for FDA approval. Possible beneficial outcomes from the use of CEUS may include, but are not limited to, the following:

- Improved characterization of focal liver masses with CEUS imaging, such as to determine whether a focal liver mass is benign or malignant, compared with nonCEUS imaging.

- Improved ability to detect focal liver masses with CEUS imaging.

- A reduction in referral for further workup using other imaging or diagnostic procedures.

- Assessment of therapeutic response.

\section{Examination Procedures}

A. The contrast agent should be prepared and administered according to the manufacturer's recommendations.

B. Equipment should be quality controlled and capable of performing CEUS imaging as described in section III, "Equipment Criteria and Variables."

C. Scans should be performed by qualified personnel as described in section $\mathrm{V}$, "Training." 
D. Baseline (noncontrast) ultrasound imaging should include representative images of the liver and any visible hepatic lesions.

E. Contrast-enhanced ultrasound image data should be acquired and stored as a real-time digital data set from before contrast agent injection until achievement of the diagnostic effect as determined for each agent/protocol.

\section{Equipment Criteria and Variables}

A. Contrast studies of the liver require contrast-specific imaging modes that operate at a low mechanical index (MI).

B. The trial protocol should specify the performance requirements of high-end systems for the study.

C. Manufacturers of ultrasound equipment have products with specifically designed presets for CEUS imaging of the liver, and these should be used.

D. Machine presets should include system parameters as well as settings that can be controlled by the user. Careful limits should be placed on the ability of the user to adjust controls once the preset has been selected. Considerations for specific settings should include the following:

1. Nonlinear Contrast Imaging Mode. As real-time imaging is a key requirement for liver imaging, a low-MI mode is needed that does not disrupt the contrast microbubbles. The mode detects the echo returning from the bubbles and suppresses the echoes from the tissue containing them. Nonlinear modes that meet these requirements usually employ multiple pulses, which are modulated in phase and/or amplitude. These schemes go under various commercial names. A machine that does not offer such a mode is not suitable for contrast imaging. Examples of suitable modes in some systems currently on the market are listed in "Appendix B."

2. Available Transducers. The appropriate transducer for contrast imaging of the liver must be specified in the protocol.
3. Mechanical Index. The MI should be maintained at a level that minimizes microbubble loss in the anatomic region of interest. The actual exposure of the agent to the ultrasound beam varies with anatomic conditions, so the fixed setting of MI provided by the preset should be used as a starting point. The displayed MI is an estimate only; actual microbubble loss is determined by factors other than the MI alone. Therefore, the appropriate MI setting should be determined separately for each ultrasound scanner model and the body habitus of each specific patient. In general, the lowest MI consistent with a successful examination should be used.

4. Operating Frequency. The operating frequency for a given machine should be specified in the protocol. In general, contrast-specific nonlinear imaging modes are more sensitive at lower operating frequencies, which also offer better penetration but lower spatial resolution. Thus, sensitivity to the agent is added to the trade-off between resolution and penetration.

5. Multiple Modes. The user must be able to view both the noncontrast and contrast images because contrast-specific imaging modes suppress the echo from tissue. Machines that offer simultaneous noncontrast and contrast-specific images are preferred. The noncontrast image MI should be controlled so that no inadvertent microbubble loss occurs.

6. Line Density/Frame Rate. In general, the lower the exposure of microbubbles to ultrasound, the lower the rate of microbubble loss. Reducing line density and frame rate to the minimum consistent with diagnostic interpretation is recommended. Zoom or magnification modes should not be used.

7. Focal Zones. Focal zone settings should be set according to manufacturers' instructions and not changed during an examination. 
8. Initial Gain. The initial gain setting, if not provided automatically by the machine, should show system noise in the far field of the contrast image.

9. Data Storage. The machine should be capable of capturing real-time image data, preferably continuously, for the duration of the contrast enhancement (at least 3 minutes is required). Storage to a local or network device is required.

E. Trial designers should work with system manufacturers to standardize machine presets for each trial.

F. Contrast-enhanced ultrasound modes should be well documented, with setup algorithms and work flow guides (see "Appendix B"). A step-by-step procedure for use of the system during the examination should be specified.

G. While the technology for CEUS modes may continue to evolve, it is recommended that the technology for an individual trial should not be changed once the trial begins.

\section{Safety}

In medical procedures, considerations should always be given to comparing the risks with the benefits to the patient. Clinical utility from improved diagnostic image information has been demonstrated with ultrasound contrast agents. Approval for the use of such agents in the United States and internationally has resulted in numerous examinations using a variety of agents (millions of doses). The general safety record of ultrasound contrast agents has been quite good, as discussed below. It is the responsibility of the ultrasound community to help maintain the safety record of ultrasound imaging and determine any risks associated with the methods used. Several aspects need to be considered:

\section{General Safety Record of Ultrasound Imaging}

Medical ultrasound imaging has been used since the 1950s. Since its introduction, this modality has been considered one of the safest methods of medical imaging. As indicated by the general clinical safety statement from the AIUM (see
"Clinical Safety" at http://www.aium.org/ publications/statements/statements.asp), there are no confirmed harmful biological effects in patients from exposure to ultrasound energy at levels commonly used for diagnostic imaging. The possibility exists that biological effects may be identified in the future, but the benefits of the prudent use of ultrasound outweigh the risk, if any, that may be present. Although this statement has largely been considered in terms of conventional non-CEUS imaging, it is important to remember that the same assessment should be made when weighing benefits and risks in clinical CEUS imaging. In the absence of specific evidence of risk and with demonstrated patient benefit, the prudent use of contrast agents should be appropriate. Additionally, when the use of a contrast agent provides significant diagnostic information, not performing such a procedure may also increase medical risk to the patient.

\section{Potential for Bioeffects}

The currently approved ultrasound contrast agents use microbubbles to produce the added contrast provided by the agent. Contrast agents are a relatively new addition to ultrasound imaging, and identifying potential risks is an ongoing process. There is a wealth of theoretical and experimental knowledge about the interactions of microbubbles and ultrasound energy. This knowledge results from a considerable number of investigations on bubble-ultrasound interactions, including cavitation activity that can have enough energy to induce bioeffects.

There have been several studies in vitro and in animal models that have shown potential bioeffects. Many of these animal studies have been related to ultrasound exposure of cardiac tissue in the presence of microbubble contrast agents. ${ }^{3-10}$ One early study in humans reported induction of premature ventricular contractions (PVCs) using a high MI and end-systolic triggering. ${ }^{11}$ However, other studies using different exposure conditions did not find an increased frequency of PVCs. ${ }^{12-15}$ The induction of PVCs in humans appears to require a relatively high MI, imaging at a specific point in the cardiac cycle, and substantial contrast agent filling of the cardiac tissue. 
In terms of noncardiac studies, some animal studies have been positive for effects, ${ }^{7,16-19}$ but the relationship to the clinical situation has not been clearly established. Of particular relevance to cancer imaging, 1 study ${ }^{20}$ did not find an enhancement of metastatic spread in mouse melanoma tumors using diagnostic levels of ultrasound in the presence of contrast agents. The identification of any potential biological effect must be placed in context. The likelihood of the effect in the clinical situation and the clinical significance of such effects should be assessed.

To the credit of the ultrasound community, there have been very active researchers who have carefully considered mechanisms for biological effects of ultrasound. These individual researchers and collective activities such as those of the Bioeffects Committee of the AIUM have helped identify potential effects that have been explored further. These efforts should continue as part of maintaining the established reputation of diagnostic ultrasound as safely practiced in medicine. The prudent use of ultrasound and CEUS imaging, including the application of the ALARA (as low as reasonably achievable) principle, should continue to the benefit of patients.

\section{Safety of CEUS Imaging}

It has been recognized for years that there are finite risks associated with the use of contrast media in medical imaging. For example, the adverse reaction rates of intravenous CT contrast agents are $4 \%$ to $12 \%$ for ionic contrast and $1 \%$ to $3 \%$ for nonionic. ${ }^{21}$ For ultrasound contrast agents, the most common effects are headache, a warm sensation, and flushing, all of which resolve in a short time. More unusual events such as nausea, dizziness, chills, altered taste, and chest pain occur in $0 \%$ to $5 \%$ of patients at a rate similar to that seen in placebo groups. ${ }^{22}$ Note that in postmarketing surveillance, the serious adverse event rate for gadolinium diethylenetriaminepentaacetic acid in MR imaging was found to be 1 to 2 per $100,000 .^{23}$ Considering the most extreme effects, the death rate from intravenous CT contrast agents is 1 to 3 per $100,000(0.002 \%) .{ }^{21}$ Only 3 deaths in approximately 160,000 doses were reported in conjunction with current ultrasound contrast agent use, all associated with 1 agent. However, continued use of this agent with changes in contraindications has yielded no additional fatalities, and debate remains as to the role of the agent in the previous deaths. No deaths have been attributed to the other agents in clinical use in the United States and elsewhere. The total number of doses for all ultrasound contrast agents combined is now almost 2 million. Therefore, the death rate associated with ultrasound contrast agents is likely significantly less than that from CT contrast agents.

Obviously these are investigations of commonly found adverse effects, and further study would be needed to follow any potential adverse effects unique to ultrasound contrast agents. However, the general safety profile is as good as or better than that of other imaging agents that are routinely used and currently deemed as acceptable risks given the benefits to the patient.

\section{Guidance for Clinical Trials}

As with any ultrasound procedure, the ALARA principle should be followed; that is, one should use only the ultrasound output power level and contrast agent dose needed for diagnostic efficacy. In terms of ultrasound exposure, the advent of low-MI imaging has significantly reduced the requisite pulse amplitude for optimal contrast imaging. Information from bioeffects studies in animals has generally found a threshold for effects at an MI of approximately 0.4 , generally higher than those typically used for low-MI imaging. This might serve as some guidance in CEUS imaging studies; however, again the specific need for higher output levels to achieve diagnostic information may be justified. For example, higher levels of acoustic power may be required on the basis of patient factors such as obesity or a deeply located lesion or if the contrast is to be eliminated from the imaging field to measure contrast flow dynamics into a lesion. In any case, the lowest level of acoustic output necessary has always been a guiding principle in medical ultrasound, and this is unchanged when considering the use of CEUS imaging.

The lack of availability of CEUS imaging for noncardiac applications in the United States denies patients a beneficial procedure, resulting in other forms of risk. 


\section{Training}

The AIUM recognizes the importance of proper qualifications of investigators and blinded readers who participate in clinical trials of CEUS. Proper experience and training are important for ultrasound imaging professionals to participate in clinical trials using ultrasound contrast agents. Although the use of contrast media requires unique skills that may not be possessed by many professionals in the United States, these skills are not difficult to learn provided the professionals have a minimum level of knowledge and experience in clinical ultrasound applications. Participation in such a program is intended to ensure a standard skill set for the purposes of the clinical trial and is not necessary after contrast agent approval, although appropriate training should be obtained by those performing clinical CEUS.

Training cases are recommended before enrolling subjects. In addition, qualified study personnel familiar with both imaging technology and contrast agent procedures should serve as onsite monitors to ensure the quality of patient examinations. Two training program models are shown in "Appendix C."

\section{Conclusion}

The AIUM believes that implementation of these recommendations will decrease variance among sites and increase the likelihood of successful clinical trials.

\section{References}

1. American Institute of Ultrasound in Medicine. Mechanical bioeffects from diagnostic ultrasound: AIUM consensus statements. J Ultrasound Med 2000; 19:69-72.

2. American Institute of Ultrasound in Medicine. American Institute of Ultrasound in Medicine Consensus Conference on Bioeffects of Diagnostic Ultrasound, 2005. J Ultrasound Med. In press.

3. Killam AL, Greener Y, McFerran BA, et al. Lack of bioeffects of ultrasound energy after intravenous administration of FS069 (Optison) in the anesthetized rabbit. J Ultrasound Med 1998; 17:349-356.

4. Ay T, Havauz X, Van Camp G, et al. Destruction of contrast microbubbles by ultrasound effects on myocardial function, coronary perfusion pressure and microvascular integrity. Circulation 2001; 104:461-466.
5. Chen S, Kroll MH, Shohet RV, Frenkel P, Mayer SA, Grayburn PA. Bioeffects of myocardial contrast microbubble destruction by echocardiography. Echocardiography 2002; 19:495-500.

6. Li P, Cao LQ, Dou CY, Armstrong WR, Miller DL. Impact of myocardial contrast echocardiography on vascular permeability: an in vivo dose response study of delivery mode, ultrasound power and contrast dose. Ultrasound Med Biol 2003; 29:1341-1349.

7. Kobayashi N, Yasu T, Yamada S, et al. Influence of contrast ultrasonography with perflutren lipid microspheres on microvessel injury. Circ J 2003; 67:630-636.

8. Li P, Armstrong WR, Miller DL. Impact of myocardial contrast echocardiography on vascular permeability: comparison of three different contrast agents. Ultrasound Med Biol 2004; 30:83-91.

9. Miller DL, Li P, Armstrong WF. The effect of time and of vasoactive drugs on capillary leakage induced during myocardial contrast echocardiography. Echocardiography 2004; 21:125-132.

10. Miller DL, Li P, Gordon D, Armstrong WF. Histological characterization of microlesions induced by myocardial contrast echocardiography. Echocardiography 2005; 22:25-34.

11. Van der Wouw P, Brauns AC, Bailey SE, Powers JE, Wilde $A A$. Premature ventricular contractions during triggered imaging with ultrasound contrast. J Am Soc Echocardiogr 2000; 13:288-294.

12. Borges AC, Walde $T$, Reibis RK, et al. Does contrast echocardiography with Optison induce myocardial necrosis in humans? J Am Soc Echocardiogr 2002; 15:1080-1086.

13. Raisinghani A, Wei KS, Crouse L, et al. Myocardial contrast echocardiography (MCE) with triggered ultrasound does not cause premature ventricular complexes: evidence from PB127 MCE studies. J Am Soc Echocardiogr 2003; 16: 1037-1042.

14. Timperley J, Mitchell AR, Thibault $\mathrm{H}$, Mirza $\mathbf{I H}$, Becher $\mathrm{H}$. Safety of contrast dobutamine stress echocardiography: a single center experience. J Am Soc Echocardiogr 2005; 18: 163-167.

15. Tsutsui JM, Elhendy A, Xie F, O'Leary EL, McGrain AC, Porter TR. Safety of dobutamine stress real-time myocardial contrast echocardiography. J Am Coll Cardiol 2005; 45: 1235-1242.

16. Miller DL, Gies RA. Gas-body-based contrast agent enhances vascular bioeffects of $1.09 \mathrm{MHz}$ ultrasound on mouse intestine. Ultrasound Med Biol 1998; 24:1201-1208.

17. Kobayashi N, Yasu T, Yamada S, et al. Endothelial cell injury in venule and capillary induced by contrast ultrasonography. Ultrasound Med Biol 2002; 28:949-956.

18. Wible JH Jr, Galen KP, Wojdyla JK, Hughes MS, Klibanov AL, Brandenburger GH. Microbubbles induce renal hemorrhage when exposed to diagnostic ultrasound in anesthetized rats. Ultrasound Med Biol 2002; 28:1535-1546.

19. Shigeta K, Itoh K, Ookawara S, Taniguchi N, Omoto K. Endothelial cell injury and platelet aggregation induced by contrast ultrasonography in the rat hepatic sinusoid. J Ultrasound Med 2004; 23:29-36. 
20. Miller DL, Dou C. Contrast-aided diagnostic ultrasound does not enhance lung metastasis in a mouse melanoma tumor model. J Ultrasound Med 2005; 24:349-354.

21. Cochran ST. Anaphylactoid reactions to radiocontrast media. Curr Allergy Asthma Rep 2005; 5:28-31.
22. Jakobsen JÅ, Oyen R, Thomsen HS, Morcos SK, Members of the Contrast Media Safety Committee of the European Society of Urogenital Radiology. Safety of ultrasound contrast agents. Eur Radiol 2005; 15:941-945.

23. Niendorf HP, Haustein J, Cornelius I, Alhassan A, Claus W. Safety of gadolinium-DTPA: extended clinical experience. Magn Reson Med 1991; 22:222-228.

\section{Appendix A: Bibliography of the World Literature on CEUS}

\section{Overview}

Blomley MJ, Cooke JC, Unger EC, Monaghan MJ, Cosgrove DO. Microbubble contrast agents: a new era in ultrasound. BMJ 2001; 322:1222-1225.

Harvey CJ, Blomley MJ, Eckersley RJ, Cosgrove DO. Developments in ultrasound contrast media. Eur Radiol 2001; 11:675-689.

\section{Characterization}

Albrecht T, Oldenburg A, Hohmann J, et al. Imaging of liver metastases with contrast-specific low-Ml real-time ultrasound and SonoVue. Eur Radiol 2003; 13(suppl 3):N79-N86.

Burns PN, Wilson SR. Enhancement patterns of focal liver masses on contrast imaging: concordance of US with CT and MR imaging. Radiology 2006; 242:162-174.

Quaia E, Calliada F, Bertolotto M, et al. Characterization of focal liver lesions with contrast-specific US modes and a sulfur hexafluoride-filled microbubble contrast agent: diagnostic performance and confidence. Radiology 2004; 232:420-430.

Solbiati L, Cova L, lerace L. Specificity of contrast-enhanced harmonic sonography for characterization of solid, incidentally detected focal liver lesions. Ultrasound Med Biol 2003; 29(suppl):S23.

Wilson S, Burns P. An algorithm for the diagnosis of focal liver masses using microbubble contrast-enhanced pulse inversion sonography. AJR Am J Roentgenol 2006; 186:1401-1412.

\section{Impact}

Cosgrove D, Blomley M. Liver tumors: evaluation with contrast-enhanced ultrasound. Abdom Imaging 2004; 29:446-454.

Leen $\mathrm{E}$. The role of contrast-enhanced ultrasound in the characterisation of focal liver lesions. Eur Radiol 2001; 11(suppl 3):E27-E34.

Leen E, Ceccotti P, Moug SJ, et al. Potential value of contrast-enhanced intraoperative ultrasonography during partial hepatectomy for metastases: an essential investigation before resection? Ann Surg 2006; 243:236-240.

Leen E, Horgan P. Ultrasound contrast agents for hepatic imaging with nonlinear modes. Curr Probl Diagn Radiol 2003; 32:66-87.

Leen E, Moug SJ, Horgan P. Potential impact and utilization of ultrasound contrast media. Eur Radiol 2004; 14(suppl 8):16-24.

Lencioni R. Impact of European Federation of Societies for Ultrasound in Medicine and Biology (EFSUMB) guidelines on the use of contrast agents in liver ultrasound. Eur Radiol 2006; 16:1610-1613.

Wink MH, Wijkstra H, De La Rosette JJ, Grimbergen CA. Ultrasound imaging and ultrasound contrast agents: a safe alternative to MRI? Minim Invasive Ther Allied Technol 2006; 15:93-100.

\section{Detection}

Albrecht T, Blomley MJ, Burns PN, et al. Improved detection of hepatic metastases with pulse-inversion US during the liver-specific phase of SHU 508A: multicenter study. Radiology 2003; 227:361-370.

Blomley MJ, Albrecht T, Cosgrove DO, et al. Improved imaging of liver metastases with stimulated acoustic emission in the late phase of enhancement with the US contrast agent SH U 508A: early experience. Radiology 1999; 210:409-416.

Hohmann J, Albrecht T, Hoffmann CW, Wolf KJ. Ultrasonographic detection of focal liver lesions: increased sensitivity and specificity with microbubble contrast agents. Eur J Radiol 2003; 46:147-159.

Quaia E, D'Onofrio M, Palumbo A, Rossi S, Bruni S, Cova M. Comparison of contrast-enhanced ultrasonography versus baseline ultrasound and contrast-enhanced computed tomography in metastatic disease of the liver: diagnostic performance and confidence. Eur Radiol 2006; 16:1599-1609. 


\section{Appendix B: Work Flow Examples}

The following manufacturers have provided current examples of work flow sequences for the use of CEUS: GE Healthcare (Milwaukee, WI), Philips Medical Systems (Bothell, WA), and Siemens Medical Solutions (Mountain View, CA). Work flow sequences from other manufacturers will be added as they become available. Draft summaries are below.

\section{Contrast Work Flow for Coded Contrast Imaging, GE LOGIQ 9, 7, and 5 (Draft)}

\begin{tabular}{|c|c|}
\hline Select New Patient control on console & Enter patient information; select Abdominal preset; exit \\
\hline Select transducer & $\begin{array}{l}\text { System will automatically default to factory- or user- } \\
\text { programmed image parameter settings for a routine exam }\end{array}$ \\
\hline Select Contrast control on console & Contrast menu appears on touch panel \\
\hline Select TruAgent Detection on touch panel & $\begin{array}{l}\text { System will automatically default to factory- or user- } \\
\text { programmed low-Ml contrast-specific image parameter } \\
\text { settings, including: } \\
\text { - Operating Frequency } \\
\text { - Acoustic Output } \\
\text { - Gain } \\
\text { - Dynamic Range } \\
\text { - Line Density } \\
\text { - Edge Enhance } \\
\text { - Pulse Repetition Integral } \\
\text { - Focal Zone number and position } \\
\text { - Gray Map } \\
\text { - Dual View Imaging mode }\end{array}$ \\
\hline \multicolumn{2}{|l|}{$\begin{array}{l}\text { Adjust gain and focal zone position as } \\
\text { necessary }\end{array}$} \\
\hline $\begin{array}{l}\text { Select Contrast Clock on touch panel on } \\
\text { agent injection }\end{array}$ & Contrast timer displays on monitor \\
\hline $\begin{array}{l}\text { Select Print } 1 \text { on console to store loops } \\
\text { or still images }\end{array}$ & $\begin{array}{l}\text { System will store raw DICOM image or loop to system } \\
\text { hard drive }\end{array}$ \\
\hline Select End Exam on exam completion & $\begin{array}{l}\text { System displays all recorded images and loops, ends exam, } \\
\text { and resets for next patient entry }\end{array}$ \\
\hline
\end{tabular}


Appendix B (continued): Work Flow Examples

\section{Contrast Work Flow for Philips iU22 (Draft)}

\begin{tabular}{|c|c|c|}
\hline Enter patient demographics & Press Patient Data key & $\begin{array}{l}\text { System provides screen menu for } \\
\text { patient data entry }\end{array}$ \\
\hline Cine loop capture length & Setups Print/Network tab & $\begin{array}{l}\text { Set prospective capture length to } \\
3 \text { minutes (only needs to be set } \\
\text { once) }\end{array}$ \\
\hline $\begin{array}{l}\text { Select transducer (or select exam } \\
\text { first) }\end{array}$ & Select Contrast Gen preset & $\begin{array}{c}\text { System sets parameters for low-Ml } \\
\text { B-mode for preinjection imaging }\end{array}$ \\
\hline Invoke contrast mode & $\begin{array}{l}\text { Press Contrast Touch Panel } \\
\text { (TP) button }\end{array}$ & $\begin{array}{l}\text { - System activates low-Ml contrast } \\
\text { agent imaging } \\
\text { - System sets transmit frequency/ } \\
\text { line density across sector } \\
\text { - System sets transmit focus to } \\
\text { encompass depth of field } \\
\text { - System sets acoustic power to } \\
\text { Ml } 0.07 \\
\text { - System sets initial gain value } \\
\text { - System sets persistence value } \\
\text { - System sets gray map value } \\
\text { - System sets Xres values } \\
\text { - System sets compression } \\
\text { - System sets 2-dimensional pulse } \\
\text { repetition frequency }\end{array}$ \\
\hline Administer agent & $\begin{array}{l}\text { Press Contrast Timer TP } \\
\text { button }\end{array}$ & $\begin{array}{l}\text { System time stamps each frame in } \\
\text { system with time since injection } \\
\text { for viewing in review }\end{array}$ \\
\hline Record clip & Press Capture & $\begin{array}{l}\text { System simultaneously records } \\
\text { DICOM and native data clips of } \\
\text { up to } 3 \text { minutes }\end{array}$ \\
\hline Stop recording clip & Press Capture key & System stops clip store \\
\hline Terminate time stamp & Press Contrast Timer & System stops rolling timer \\
\hline To change transmit frequency & $\begin{array}{l}\text { Toggle 2D Opt (Pen, Gen, } \\
\text { Res) }\end{array}$ & $\begin{array}{l}\text { System sets new transmit } \\
\text { frequency and recalculates Ml } \\
\text { and Ml at the focus (MIF) values }\end{array}$ \\
\hline To display live dual imaging & Press Contrast Side/Side & $\begin{array}{l}\text { System displays agent image on } \\
\text { one side and tissue image on the } \\
\text { other side }\end{array}$ \\
\hline $\begin{array}{l}\text { To do region of interest (ROI) } \\
\text { analysis or microvascular } \\
\text { imaging (MVI) processing } \\
\text { (to view bubble outline of } \\
\text { vasculature) }\end{array}$ & $\begin{array}{l}\text { Press QLab, in review; } \\
\text { then press ROI or MVI }\end{array}$ & Select ROI or MVI \\
\hline
\end{tabular}


Appendix B (continued): Work Flow Examples

\section{Contrast Work Flow for Contrast Pulse Sequencing (CPS), Siemens Sequoia System}

\begin{tabular}{lc}
\hline Enter patient demographics & Press Begin Exam key \\
\hline Select exam (or select & Press Exam key; select CPS \\
transducer first) & Abdomen from Exam list \\
Select transducer (or select & Press Transducer key; select \\
exam first) & transducer from soft key
\end{tabular}

System provides screen menu for patient data entry

exam first)

- System sets parameters for standard B-mode preinjection imaging

- System sets standard B-mode imaging presets

- Exception: system sets digital clip capture for 8 minutes

Invoke contrast mode Press Cadence key

- System activates low-MI CPS agent imaging

- System sets to lowest transmit frequency

- System sets transmit focus to encompass depth of field

- System sets acoustic power to -21 dB and calculates corresponding MI and MIF

- System sets initial gain value

- System sets persistence value

- System sets post processing map value

- System sets enhancement value

- System sets Delta value

- System sets agent-only display

\begin{tabular}{|c|c|c|}
\hline Equalize image brightness & Press TEQ key & $\begin{array}{l}\text { - System sets lateral and axial gain } \\
\text { - System detects and subtracts } \\
\text { background noise }\end{array}$ \\
\hline Administer agent & Press Stopwatch key & $\begin{array}{l}\text { System time stamps current time of day } \\
\text { and initiates rolling stopwatch }\end{array}$ \\
\hline Record clip & Press Clip Store key & $\begin{array}{l}\text { System simultaneously records } \\
\text { DICOM and QuickTime clips of } \\
\text { up to } 8 \text { minutes }\end{array}$ \\
\hline Stop recording clip & Press Clip Store key & System stops clip store \\
\hline Terminate time stamp & $\begin{array}{l}\text { Press Stopwatch Stop/Reset } \\
\text { soft key }\end{array}$ & $\begin{array}{l}\text { System stops rolling stopwatch } \\
\text { and zeros stopwatch }\end{array}$ \\
\hline To change transmit frequency & Toggle MultiHz key & $\begin{array}{l}\text { System sets new transmit frequency } \\
\text { and recalculates MI and MIF values }\end{array}$ \\
\hline To display live dual imaging & Press Dual key & $\begin{array}{l}\text { System displays agent image on one } \\
\text { side and tissue image on the other side }\end{array}$ \\
\hline $\begin{array}{l}\text { To switch from agent-only to } \\
\text { mix or tissue-only display }\end{array}$ & Toggle Balance key & $\begin{array}{l}\text { System displays mixed agent and } \\
\text { tissue image or tissue-only image } \\
\text { or agent-only image }\end{array}$ \\
\hline
\end{tabular}




\section{Appendix C: A CEUS Imaging Training Program}

Two concepts for training programs have been developed as described in the outline below. The training programs are generic (ie, they are not designed to be used for a specific contrast product), but they could be easily modified to incorporate any unique or product-specific requirements related to a given ultrasound contrast agent (eg, preparation, doses, etc). Once the trainees complete the training program(s), they would be required to take an examination that would indicate their level of competency in CEUS imaging studies.

\section{Participants}

Proof of adequate experience and/or participation in these training programs is required for all ultrasound imaging professionals that intend to perform CEUS examinations as part of clinical trials and those that will function as "blinded readers" of CEUS studies performed in other laboratories.

It is suggested that proof of adequate experience and/or participation in a training program also be required for clinical representatives (eg, application specialists, etc) of pharmaceutical and ultrasound system vendors that will be involved in the clinical trials.

Similar training programs could be used for professionals that desire CEUS training after CEUS is approved for clinical trials.

\section{Training Programs}

I. Two training programs could be developed.

A. One program would be designed for physicians who intend to be "blinded readers" for CEUS clinical trials.

B. One program would be designed for physicians and sonographers who intend to participate in CEUS clinical trials.

II. Applicants would be required to possess prerequisite qualifications to participate.

A. For physicians, board certification and a minimum of 5 years' experience in ultrasound would be required.

B. For sonographers, American Registry for Diagnostic Medical Sonography certification in abdominal ultrasound would be required.

C. Laboratory accreditation as a requirement may be considered.

D. Qualified individuals could participate in either or both training programs.

III. Training programs would be generic in terms of the contrast media (ie, they would not be designed for a specific contrast product).

IV. Applicants would be required to take a pretest before beginning the training programs and a posttest after completion of the programs.

A. Use of pretests and posttests permits analyses of the level of knowledge that trainees obtain by completing the training programs as well as the adequacy of the educational programs.

V. Relevant reference materials would be made available to applicants.

A. Materials may include published reports, book chapters, European contrast agent guidelines, white papers, etc.

VI. Training would include the following:

A. Didactic training for all applicants.

B. Hands-on training for applicants who intend to perform CEUS clinical trials.

C. Didactic lectures would include the following:

1. Physical principles of CEUS, including instrumentation and scanning techniques. 


\section{Appendix C (continued): A CEUS Imaging Training Program}

2. A review of the relevant anatomy, physiology, and pathology as it pertains to CEUS for liver applications.

3. Pitfalls, limitations, safety, and other relevant issues.

4. Patient/subject selection.

D. Hands-on training would use a wet lab with an animal model and all ultrasound systems that are deemed appropriate for CEUS trials.

1. Trainees would perform CEUS studies using equipment that they would use in their own lab with supervision by CEUS-experienced nurses, sonographers, and physicians.

2. Contrast media manufacturers' representatives would also be involved in the wet lab training (for a given agent).

VII. Case reviews would be performed.

A. Before the trainees could perform or interpret CEUS protocol studies, a specified number of CEUS teaching cases would be reviewed.

1. The training cases would be in real time on DVD (or other video format).

2. Trainees would review the cases and complete a questionnaire that would include a description of the features of the CEUS studies, diagnostic impressions/differentials, limitations, level of suspicion, final assessment, etc.

3. The trainees' responses on the forms would be compared with those of a panel of experts.

VIII. Trainees who successfully complete the formal training programs, case reviews, and posttests would be "signed off" as being competent to perform and/or interpret CEUS protocol examinations.

A. Trainees who do not successfully complete either the formal training and/or the required number of case reviews would be required to continue the training process with emphasis on their areas of weakness as determined by the posttest and/or case review questionnaires. They would then be required to retake the posttest until successful. 